Palavras chave:

Cerrado Típico

Savanas

Sistema Aquífero Urucuia

Histórico:

Recebido 06/06/2013

Aceito 14/09/2015

Keywords:

Typical Cerrado

Savannas

Urucuia Aquifer System

Correspondência: camiflorestal@gmail.com
Camila Paula de Oliveira', Márcio Rocha Francelino', Vinícius Costa Cysneiros', Fernando Canto de Andrade', Micael Cortopassi Booth'

\section{COMPOSIÇÃO FLORÍSTICA E ESTRUTURA DE UM CERRADO SENSU STRICTO NO OESTE DA BAHIA}

RESUMO: Neste trabalho foi caracterizada a florística e a estrutura de um Cerrado sensu stricto localizado no município de Jaborandi, oeste baiano. Para tanto, foram amostrados todos os indivíduos com diâmetro à $30 \mathrm{~cm}$ da altura da base $\left(D_{30 \mathrm{~cm}} \geq 5 \mathrm{~cm}\right)$ presentes em área amostral de dois hectares composta por 20 parcelas de $1000 \mathrm{~m}^{2}(20 \mathrm{~m} \times 50 \mathrm{~m})$. Foram encontrados 2054 indivíduos (1027 ind $\cdot \mathrm{ha}^{-1}$ ), com área basal de 8,24 $\mathrm{m}^{2} \cdot \mathrm{ha}^{-1}$, distribuídos em 58 espécies pertencentes a 42 gêneros e 24 famílias. A família Fabaceae apresentou maior número de espécies ( I2), seguida de Myrtaceae (8) e Vochysiaceae (5). Os descritores fitossociológicos avaliados revelaram uma comunidade caracterizada pela presença de poucas espécies dominantes, onde a maioria pode ser considerada como pouco comuns e raras. As estruturas diamétrica e da altura evidenciaram uma vegetação equilibrada e composta predominantemente por indivíduos de pequeno porte, onde a riqueza é relevante, assim como a diversidade local, que foi estimada em 2,95 nats.ind ${ }^{-1}$ pelo índice de Shannon-Wiener.

\section{STRUCTURE AND FLORISTIC OF THE CERRADO SENSU STRICTO IN WEST OF BAHIA}

ABSTRACT: In this study it was characterized the structure and floristic of the cerrado sensu stricto located in Jaborandi, a city of western Bahia State. All individuals were sampled based on diameter height $\left(D_{30 \mathrm{~cm}} \geq 5 \mathrm{~cm}\right)$ present in a sample area of two hectares comprising 20 plots of $1000 \mathrm{~m}^{2}(20 \mathrm{~m} \times 50 \mathrm{~m})$. Individuals were found in total of 2054 (I027 ind.ha- ${ }^{-1}$ ), on a basal area of $8.24 \mathrm{~m}^{2} \cdot \mathrm{ha}^{-1}$ and 58 species belonging to 42 genera and 24 families. Fabaceae had the largest number of species (12), followed by Myrtaceae (8) and Vochysiaceae (5). The phytosociological descriptors evaluated, have shown a community characterized by the presence of a few dominant species, where most can be regarded as unusual and rare. The diametrical structure and height presented a balanced vegetation, predominantly composed by small individuals. Here, richness is relevant, as well as local diversity, which was estimated at 2.95 nats.ind ${ }^{-1}$ by the Shannon-Wiener index. 


\section{INTRODUÇÃO}

Considerada a savana tropical mais biodiversa do mundo (KLINK; MACHADO, 2005), o Cerrado se destaca pela marcante heterogeneidade de habitats, que comportam elevado número de elementos endêmicos da fauna e principalmente da flora, colocando-o entre um dos hotspots mais ameaçados do planeta (MITTERMEIER et al., 2005). Sua vegetação compreende um verdadeiro mosaico de ambientes, onde estão presentes diversos tipos de ecossistemas, que variam desde formações abertas, subdivididas entre formações campestres e savânicas, até fisionomias florestais (RIBEIRO; WALTER, 2008). A forma mais extensa, o Cerrado sensu stricto, ocupa aproximadamente $65 \%$ da área geográfica original do bioma, onde no restante, diversos outros tipos fitofisionômicos dividem a paisagem (MARIMOM JUNIOR; HARIDASAM, 2005).

Nesta fitofisionomia, a expressiva diversificação florística é proporcionada pela distribuição espacial das espécies em mosaicos, fazendo com que comunidades relativamente próximas apresentem características florísticas e estruturais distintas, o que lhe confere elevada diversidade beta (FELFILI et al., 2004). Ocorrendo geralmente associado a solos profundos e relevo plano, o Cerrado sensu stricto se caracteriza pelo baixo porte de seu estrato arbóreo tortuoso e pouco denso (RIBEIRO; WALTER, 2008), o que torna sua supressão facilitada, que aliada ao desejo de expansão da agropecuária neste relevo favorável, coloca esta fitofisionomia, a mais representativa e extensa do bioma, sobre forte pressão antrópica.

No oeste da Bahia, a implantação de uma dinâmica agrícola moderna na região dos cerrados, certamente constituiu-se em importante avanço econômico para o estado. Por outro lado, contribuiu substancialmente para $\circ$ processo acelerado de devastação do bioma e depauperação dos recursos ambientais desta região. $O$ modelo de ocupação destas áreas, muitas vezes realizado de maneira simplista, ignora os princípios de sustentabilidade destes ambientes ao incorporar ao processo produtivo áreas fundamentais à manutenção da biodiversidade e serviços ecossistêmicos essenciais, gerando elevado prejuízo ambiental. Neste sentido, torna-se relevante estudar a estrutura e composição dos cerrados remanescentes desta região, como forma de contribuir cientificamente para o preenchimento das lacunas do conhecimento no que diz respeito aos cerrados do oeste da Bahia (MACHADO et al., 2008) e possibilitar o ordenamento destes recursos, viabilizando a adoção de práticas ambientalmente adequadas, aliadas a conservação destes ambientes, bem como subsidiar estudos para recuperação das áreas degradadas no bioma. Portanto, buscamos neste trabalho, caracterizar a florística e a estrutura do componente arbóreo de um Cerrado sensu stricto localizado no município de Jaborandi, no oeste da Bahia.

\section{MATERIAL E MÉTODOS}

\section{Caracterização da área}

A Fazenda Guará, onde o estudo foi conduzido, localiza-se no município de Jaborandi, oeste baiano, entre as coordenadas $14^{\circ} 42^{\prime} \mathrm{S}$ e $45^{\circ} 53^{\prime} \mathrm{N}$. Está totalmente inserida no bioma Cerrado, sendo quase inteiramente coberta por vegetação nativa com grande diversidade de ambientes, distribuídos em 9.569,55 ha, onde a altitude varia de $765 \mathrm{~m}$ a $943 \mathrm{~m}$, sendo o relevo predominantemente plano à suave ondulado.

Situada na unidade fisiográfica do Espigão Mestre do São Francisco (RESENDE; GUIMARÃES, 2007), compreende a área de ocorrência da Formação Urucuia (BRASIL, 1982), localizado a oeste da média bacia do rio São Francisco. Neste substrato litológico predominam arenitos quartzosos de cores variadas com granulometria variando de fina a média. Tais arenitos refletem diretamente nas características físicas e químicas dos solos da fazenda, onde a ordem mais comum é a dos Latossolos (SILVA, 20I I), predominando solos de classe textural franco-arenosa, extremamente intemperizados e de baixa fertilidade natural, além de serem fortemente drenados.

O clima regional é, de acordo com a classificação de Köppen, considerado Aw Tropical Sazonal de Inverno Seco. A precipitação média anual varia de $700 \mathrm{~mm}$ a 1.400 $\mathrm{mm}$. A temperatura média anual é da ordem de $24^{\circ} \mathrm{C}$ e a umidade relativa média anual é de cerca de $70 \%$, sendo que os valores máximos mensais ocorrem no período de novembro a maio, e os mínimos acontecem de Junho a Outubro.

Os limites da propriedade estão totalmente inseridos na área efetiva do Sistema Aquífero Urucuia (CPRM, 20I0). Além disso, a área insere-se no corredor ecológico criado para manter a conectividade entre $\circ$ Parque Nacional Grande Sertão Veredas e o Refúgio da Vida Silvestre do Oeste Baiano sendo, portanto, área prioritária para a criação de novas unidades de conservação e adoção de práticas agrícolas ambientalmente adequadas 
(INEMA, 20I4; RESENDE; GUIMARÃES, 2007).

Silva (20I I), concluiu que a fazenda apresentava menos de $2 \%$ da sua área com algum tipo de uso de caráter antrópico, sendo o restante ocupado por vegetação nativa. Conforme a autora, dentre as formações classificadas de acordo com Ribeiro e Walter (2008), o Cerrado sensu stricto típico é a fitofisionomia mais abundante, se estendendo por aproximadamente $64,33 \%$ da área total da propriedade, onde ocorre geralmente associado à Latossolos Vermelho-Amarelos, na áreas mais altas e planas, e à Neossolos Quartzarênicos próximo aos Campos limpos, no terço inferior. $\mathrm{Na}$ paisagem também estão presentes o Cerrado sensu stricto ralo, e o denso, os Campos limpos e sujos, as Veredas, Matas de galeria e o Cerradão distrófico.

\section{Amostragem, procedimentos específicos e análise dos dados}

Foi selecionada uma área de 30 ha completamente coberta por Cerrado sensu stricto. Nesta área foram alocadas 20 unidades amostrais de $1000 \mathrm{~m}^{2}(20 \mathrm{~m} \times 50 \mathrm{~m})$ (FELFILI et al., 2005), dispostas aleatoriamente, totalizando dois hectares. Em cada uma das unidades amostrais, foram listados todos os indivíduos lenhosos, assim como os do gênero Vellozia sp., que apresentaram diâmetro à $30 \mathrm{~cm}$ da altura da base igual ou superior a $5 \mathrm{~cm}\left(D_{30 \mathrm{~cm}} \geq 5 \mathrm{~cm}\right.$ ) (FELFILI et al., 2005). Os indivíduos que se bifurcaram antes da altura de medição do diâmetro, tiveram seus fustes mensurados, quando estes atenderam ao critério de inclusão estabelecido. A altura total foi estimada utilizandose uma vara graduada em centímetros. Os indivíduos tiveram material botânico coletado para confecção de exsicatas, que foram depositadas no Herbário do Departamento de Botânica da Universidade Federal Rural do Rio de Janeiro (RBR). A identificação botânica foi realizada com auxílio de bibliografia, especialistas e consultas ao Herbário do Departamento de Botânica da UFRRJ (RBR), assim como os Herbários digitais, Jabot - Banco de Dados da Flora Brasileira, disponível em: [http://jbrj.gov.b/jabot] e INCT - Herbário Virtual da Flora e dos Fungos, disponível em [http://inct.splink.org. br]. A sistematização das espécie seguiu a classificação taxonômica proposta pelo APG III (APG, 2009).

A suficiência amostral foi avaliada por análise da curva de acumulação de espécies (FELFILI et al., 20I I). A estrutura horizontal foi caracterizada por meio do cálculo dos descritores fitossociológicos absolutos e relativos de densidade, dominância e frequência das espécies, bem como o valor de importância e de cobertura (FELFILI et al., 20I I). A diversidade foi estimada pelo índice de Shannon-Wiener $\left(\mathrm{H}^{\prime}\right)$ na escala logarítmica e a equitabilidade conforme Pielou (J') (MAGURRAM, 20II). Para avaliar a estrutura diamétrica e hipsométrica, foram feitos os histogramas de frequência da distribuição dos fustes em classes de diâmetro com amplitude de quatro centímetros e em classes de altura com amplitude de $0,5 \mathrm{~m}$. Posteriormente, a distribuição diamétrica foi ajustada ao modelo exponencial negativo de Meyer e os quocientes " $q$ " De Liocurt calculados para cada classe estabelecida (MEYER, 1952; apud FELFILI; SILVA JÚNIOR, 1988).

\section{RESULTADOS E DISCUSSÃO}

\section{Suficiência amostral}

A tendência de estabilidade verificada pela curva de acumulação de espécies, revelou que metade da área amostral foi suficiente para amostrar $80 \%$ do total de 58 espécies verificadas na área (Figura I). A partir da $17^{\mathrm{a}}$ unidade amostral não ocorreram novas espécies, quando a curva se estabilizou, demonstrando eficiente amostragem da variabilidade florística local (MEDEIROS

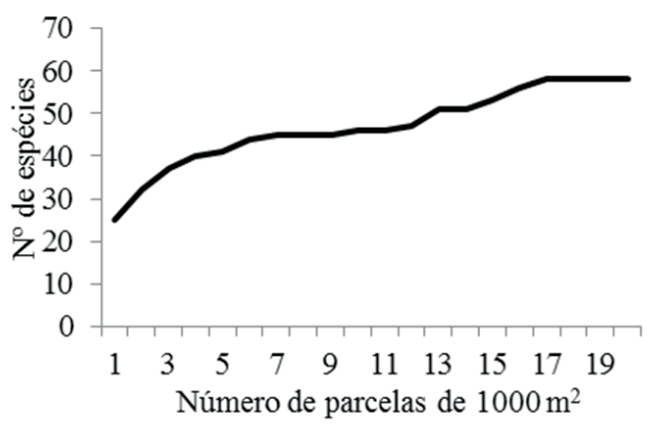

Figura I Curva de acumulação de espécies para o Cerrado sensu stricto estudado em Jaborandi, BA.

Figure I Species accumulation curve for the Cerrado sensu stricto studied in Jaborandi, BA.

et al., 2007).

\section{Composição Florística e Estrutura}

Foram amostrados 2.054 indivíduos (2.125 fustes) identificados em 58 espécies pertencentes a 42 gêneros e 24 famílias botânicas. A riqueza de espécies encontrada se aproximou do limite inferior do intervalo dos valores apresentados por Felfili et al. (2004), onde a riqueza variou de 66 a 97 espécies em seis áreas amostradas na região do Espigão Mestre do São Francisco, mesma unidade fisiográfica do presente 
estudo. Corroborando com o resultado encontrado, Assunção e Felfili (2004), identificaram 54 espécies lenhosas em um Cerrado sensu stricto no DF. Os autores enfatizaram a semelhança do padrão da comunidade estudada com áreas amostradas no Parque Nacional Grande Sertão Veredas, vizinho à área do presente estudo, e demais localidades no oeste da Bahia.

As famílias com maior número de espécies foram Fabaceae (12), Myrtaceae (8), Vochysiaceae (5), Malphigiaceae e Connaraceae (4), seguidas de Apocynaceae e Erythroxylaceae com três espécies. Outras seis famílias apresentaram duas espécies e onze famílias apenas indivíduos de uma espécie. Conforme Mendonça et al. (2008), é frequente a presença das famílias Vochysiaceae, Fabaceae e Myrtaceae nos cerrados no Brasil central.

As sete espécies mais importantes foram: Pouteria ramiflora (Mart.) Radlk, Kielmeyera coriacea Mart. \& Zucc, Pouteria torta (Mart.) Radlk, Aspidosperma tomentosum Mart., Vellozia squamata Pohl, Connarus suberosus Planch. e Salvertia convallariodora A. St.Hil., que perfizeram $52,58 \%$ do valor de importância, $65,04 \%$ da densidade total e $63,36 \%$ da dominância
(Tabela I). Exceto Pouteria torta, as demais espécies citadas são listadas por Ratter et al. (2003) dentre as espécies que ocorreram em mais de $50 \%$ do total de 376 levantamentos comparados em Cerrado sensu stricto na área core do bioma. Os elevados $\mathrm{VI}$ observados para Pouteria ramiflora e Kielmeyera coriacea ocorreram devido aos elevados percentuais de dominância e densidade respectivamente. Exceto Salvertia convallariodora, que apresentou alta dominância, as demais espécies apresentaram elevados VI devido à densidade. Pouteria ramiflora, Kielmeyera coriacea, Pouteria torta, Aspidosperma tomentosum, Connarus suberosus e Ouratea hexasperma (A.St.-Hil.) Baill tiveram frequência absoluta de $100 \%$.

As sete espécies com maiores VI representaram $12 \%$ do total de espécies amostradas. Trinta e sete espécies apresentaram $\mathrm{VI}$ inferiores a I,55\%, valor que representa menos de $10 \%$ do máximo encontrado $(15,50)$, indicando que estas espécies são pouco comuns na área de estudo (ASSUNÇÃO; FELFILI, 2004). Byrsonima sp. 2, Buchenavia tomentosa Eichler, Annona crassiflora Mart., Acosmium sp., Vochysia rufa Mart. e Vatairea macrocarpa (Benth.) Ducke, apresentaram apenas um indivíduo por hectare, podendo ser consideradas localmente raras. Aspidosperma macrocarpa

Tabela I Descritores fitossociológicos das espécies encontradas em dois hectares de Cerrado sensu stricto em Jaborandi, BA.

Table I Phytosociological descriptors of species found in two hectares of Cerrado sensu stricto in Jaborandi, BA.

\begin{tabular}{|c|c|c|c|c|c|}
\hline Espécies/Famílias & $\mathrm{N}$ & UA & $g$ & DA & $\mathrm{VI} \%$ \\
\hline Pouteria ramiflora (Mart.) Radlk./Sapotaceae & 406 & 20 & $\mathrm{I}, 8442$ & 203 & 15,50 \\
\hline Kielmeyera coriacea Mart. \& Zucc./Calophyllaceae & 306 & 20 & 1,0210 & 153 & 10,54 \\
\hline Pouteria torta (Mart.) Radlk./Sapotaceae & 165 & 20 & 0,6592 & 83 & 6,79 \\
\hline Aspidosperma tomentosum Mart./Apocynaceae & 153 & 20 & 0,4003 & 77 & 5,55 \\
\hline Vellozia squamata Pohl/Velloziaceae & 151 & 19 & 0,3909 & 76 & 5,41 \\
\hline Connarus suberosus Planch./Connaraceae & 103 & 20 & 0,3155 & 52 & 4,40 \\
\hline Salvertia convallariodora A. St.- Hil./Nochysiaceae & 47 & 17 & 0,5906 & 24 & 4,38 \\
\hline Ouratea hexasperma (A.St.-Hil.) Baill./Ochnaceae & 70 & 20 & 0,2957 & 35 & 3,78 \\
\hline Byrsonima coccolobifolia Kunth./Malphigiaceae & 57 & 18 & 0,1791 & 29 & 2,95 \\
\hline Erythroxylum deciduum A. St.-Hil./Erythroxylaceae & 58 & 17 & $0,145 \mid$ & 29 & 2,76 \\
\hline Hymenaea stigonocarpa Mart. ex Hayne/Fabaceae-Caesalpinioideae & 40 & 15 & 0,2385 & 20 & 2,70 \\
\hline Andira vermifuga (Mart.) Benth/Fabaceae-Papilionoideae & 51 & 15 & 0,1816 & 26 & 2,65 \\
\hline Vochysia tucanorum Mart./Vochysiaceae & 49 & 16 & 0,1404 & 25 & 2,52 \\
\hline Eugenia dysenterica D. C./Myrtaceae & 40 & 14 & 0,1233 & 20 & 2,16 \\
\hline Handroanthus ochraceus (Cham.) Mattos/Bignoniaceae & 32 & 15 & 0,0903 & 16 & 1,97 \\
\hline Rourea induta Planch./Connaraceae & 30 & 15 & 0,0784 & 15 & 1,89 \\
\hline Agonandra brasiliensis Miers ex Benth. \& Hook. f./Opiliaceae & 31 & 14 & 0,0839 & 16 & $\mathrm{I}, 86$ \\
\hline Kyelmeyera petiolaris Mart./Calophyllaceae & 23 & II & 0,1400 & 12 & $\mathrm{I}, 74$ \\
\hline Erythroxylum suberosum A.St.-Hil./Erythroxylaceae & 28 & 13 & 0,0607 & 14 & $\mathrm{I}, 64$ \\
\hline
\end{tabular}

Continua... 


\begin{tabular}{|c|c|c|c|c|c|}
\hline Espécies/Famílias & $\mathrm{N}$ & UA & $g$ & DA & $\mathrm{VI} \%$ \\
\hline Machaerium opacum Vogel/Fabaceae-Papilionoideae & 17 & 12 & 0,1076 & 9 & 1,58 \\
\hline Qualea parviflora Mart.Nochysiaceae & 17 & 9 & 0,1534 & 9 & $\mathrm{I}, 55$ \\
\hline Mouriri lliptica Mart. /Melastomataceae & 19 & 13 & 0,0715 & 10 & $\mathrm{I}, 54$ \\
\hline Byrsonima verbascifolia (L.) DC./Malphigiaceae & 15 & 9 & 0,0505 & 8 & 1,10 \\
\hline Caryocar brasiliense Cambess./Caryocaraceae & 16 & 4 & 0,1122 & 8 & 1,00 \\
\hline Enterolobium gummiferum (Mart.) J. F. Macbr./Fabaceae-Mimosoideae & 8 & 6 & 0,1033 & 4 & 0,98 \\
\hline Bowdichia virgilioides Kunt/Fabaceae-Papilionoideae & 8 & 5 & 0,1121 & 4 & 0,95 \\
\hline Myrocarpus fastigiatus Allemão/ Fabaceae-Caesalpinoideae & 6 & 4 & 0,1193 & 3 & 0,87 \\
\hline Strychnos pseudoquina A.St.-Hil./Loganiaceae & 5 & 4 & 0,0811 & 3 & 0,70 \\
\hline Psidium myrsinites DC./ Myrtaceae & II & 5 & 0,0367 & 6 & 0,69 \\
\hline Leptolobium dasycarpum Vog./Fabaceae - Papilionoideae & 8 & 6 & 0,0246 & 4 & 0,66 \\
\hline Heisteria ovata Benth./Olacaceae & 7 & 5 & 0,0338 & 4 & 0,61 \\
\hline Eriotheca gracilipes (K. Schum.) A. Robyns/Malvaceae & 9 & 3 & 0,0545 & 5 & 0,58 \\
\hline Tabebuia aurea (Silva Manso) Benth. \& Hook f. ex S. Moore/Bignoniaceae & 6 & 5 & 0,0231 & 3 & 0,55 \\
\hline Qualea grandiflora Mart./Nochysiaceae & 5 & 5 & 0,0224 & 3 & 0,53 \\
\hline Myrcia guianensis (Aubl.) DC/Myrtaceae & 6 & 4 & 0,0152 & 3 & 0,45 \\
\hline Stryphnodendron adstrigens (Mart.) Coville/ Fabaceae-Mimosoideae & 5 & 4 & 0,0103 & 3 & 0,41 \\
\hline Neea theifera Oerst. /Nyctaginaceae & 5 & 4 & 0,0058 & 3 & 0,39 \\
\hline Psidium pohlianum O. Berg / Myrtaceae & 4 & 4 & 0,0080 & 2 & 0,39 \\
\hline Palicourea rigida Kunth/Rubiaceae & 4 & 3 & 0,0073 & 2 & 0,31 \\
\hline Byrsonima sp. 2 /Malphigiaceae & 3 & 3 & 0,0085 & 2 & 0,30 \\
\hline Tocoyena formosa (Cham. \& Schltdl.) K. Schum / Rubiaceae & 4 & 3 & 0,0042 & 2 & 0,30 \\
\hline Buchenavia tomentosa Eichler/ Combretaceae & 2 & 2 & 0,0166 & 1 & 0,24 \\
\hline Annona crassiflora Mart./Annonaceae & 2 & 2 & 0,0116 & I & 0,22 \\
\hline Acosmium sp./ Papilionoideae & 3 & 2 & 0,0050 & 2 & 0,21 \\
\hline Vochysia rufa Mart./ Vochysiaceae & 2 & 2 & 0,0074 & I & 0,21 \\
\hline Byrsonima sp. I/ Malphigiaceae & 4 & I & 0,0159 & 2 & 0,20 \\
\hline Vatairea macrocarpa (Benth.) Ducke/Fabaceae - Papilionoideae & 2 & I & 0,0119 & I & 0,15 \\
\hline Aspidosperma macrocarpa Mart./ Apocynaceae & 1 & I & 0,0043 & I & 0,11 \\
\hline Dimorphandra mollis Benth. / Fabaceae - Caesalpinioideae & 1 & I & 0,0038 & 1 & 0,10 \\
\hline Myrcia ochroides O. Berg/Myrtaceae & 1 & I & 0,0038 & 1 & 0,10 \\
\hline Eugenia spl./Myrtaceae & 1 & I & 0,0033 & 1 & 0,10 \\
\hline Hancornia speciosa Gomes / Apocynaceae & 1 & I & 0,0033 & I & 0,10 \\
\hline Rourea sp. / Connaraceae & 1 & I & 0,0023 & 1 & 0,10 \\
\hline Tachigali paniculata Aubl. / Fabaceae - Caesalpinioideae & 1 & I & 0,0019 & 1 & 0,10 \\
\hline Aegiphila ihotzkiana Cham./Lamiaceae & 1 & I & 0,0018 & 1 & 0,10 \\
\hline Miconia pohliana Cogn./ Melastomataceae & I & I & 0,0016 & I & 0,10 \\
\hline Eugenia sp2. / Myrtaceae & 1 & I & 0,0014 & I & 0,09 \\
\hline Erythroxylum sp./ Erythroxylaceae & 1 & I & 0,0009 & 1 & 0,09 \\
\hline Total Geral & 2054 & 20 & 8,2413 & 1027 & 100 \\
\hline
\end{tabular}

Valores decrescentes em função do VI (Valor de Importância). N=Número de indivíduos da encontrados da espécie; UA= Número de unidades amostrais em que a espécie ocorre; $\mathrm{g}=$ área basal da espécie $\left(\mathrm{m}^{2} \cdot \mathrm{ha}^{-1}\right)$; $\mathrm{DA}=\mathrm{Densidade}$ absoluta da espécie (número de indivíduos $\cdot \mathrm{ha}^{-1}$ ); VI\%=valor de importância da espécie. 
Mart., Dimorphandra mollis Benth., Myrcia ochroides O. Berg, Eugenia sp.I, Hancornia speciosa Gomes, Rourea sp., Tachigali paniculata Aubl., Aegiphila ihotskiana Cham., Miconia pohliana Cogn., Eugenia sp.2 e Erythroxylum sp., apresentaram apenas um indivíduo nos dois hectares de Cerrado sensu stricto amostrados. Estas 17 espécies representaram $29 \%$ do número total de espécies encontradas na comunidade, 1,21\% do número total de indivíduos amostrados e $2,43 \%$ do VI total. Observa-se, portanto, uma vegetação caracterizada pela presença de poucas espécies dominantes, de modo que as espécies pouco comuns ou raras apresentam pequena contribuição na ocupação do espaço. Conforme Haridasam (2005), a maioria dos levantamentos realizados em Cerrado sensu stricto comprova que menos da metade de todas as espécies encontradas são responsáveis por mais de $75 \%$ do número de indivíduos e área basal da comunidade.

A diversidade florística estimada pelo índice de diversidade de Shannon-Wiener $\left(\mathrm{H}^{\prime}\right)$ foi de 2,95 nats.ind ${ }^{-1}$. Este valor se aproximou do limite inferior do intervalo de diversidade verificado para 15 áreas amostradas na Chapada Pratinha, Chapada dos Veadeiros e Espigão Mestre do São Francisco, onde o índice de Shannon variou de 3,04 a 3,73 nats·ind ${ }^{-1}$, a riqueza de 55 a 97 e o índice de Pielou de 075 a 0,88 (FELFILI et al., 1994, 2004, 2007). Por considerar a abundância das espécies na estimativa da diversidade, é provável que esta menor diversidade estimada pelo índice de Shannon no presente estudo, se deve à elevada dominância de poucas espécies na área estudada, como Kielmeyera coreacea e Pouteria ramiflora, o que se confirma pela equitabilidade estimada em 0,73 , que

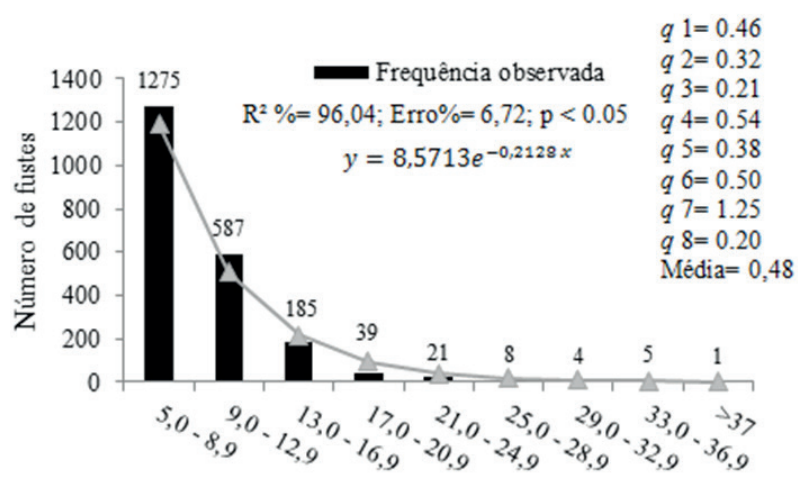

Classes de diâmetro $(\mathrm{cm})$

Figura 2 Estrutura diamétrica ajustada ao modelo exponencial negativo de Meyer e quocientes "q" De Liocurt do Cerrado sensu stricto amostrado em Jaborandi, BA.

Figure 2 Diameter structure adjusted to the negative exponential model of Meyer and Liocurt's " $q$ " quotients of Cerrado sensu stricto sampled at Jaborandi, BA. revela uma menor uniformidade na distribuição do número de indivíduos por espécies nesta vegetação.

A densidade total desta vegetação, considerandose a presença da espécie não lenhosa Vellozia squamata, foi de 1027 ind $\cdot \mathrm{ha}^{-1}$ com área basal de $8,24 \mathrm{~m}^{2} \cdot \mathrm{ha}^{-1}$. Para os indivíduos lenhosos, a densidade total foi de $95 \mathrm{I}$ ind $\cdot \mathrm{ha}^{-1} \mathrm{com}$ área basal de 7,85 $\mathrm{m}^{2} \cdot \mathrm{ha}^{-1}$. Conforme Felfili (2008) o intervalo de 400 a 1000 ind.ha ${ }^{-1}$ é considerado representativo para a vegetação lenhosa destes cerrados da região do Espigão Mestre do São Francisco onde predominam os Neossolos. Para a área basal, considera-se representativa a faixa de 3 a $8 \mathrm{~m}^{2} \cdot \mathrm{ha}^{-1}$ para os cerrados desta região.

A estrutura diamétrica da comunidade obedeceu ao padrão "J-reverso" ou exponencial negativo, com a maioria dos fustes concentrados nas três primeiras classes de diâmetro, somando $96,33 \%$ do número total de fustes amostrados (Figura 2).

Quando se observa os valores do quociente De Liocour "q" ao longo das nove classes diamétricas, verificase que a comunidade se apresenta estruturalmente irregular. Entretanto, o decréscimo uniforme na frequência de indivíduos nas classes diamétricas e o bom ajuste do modelo exponencial negativo de Meyer evidenciado pelo elevado $R^{2}(p<0,05)$, indicam estrutura diamétrica tendendo ao equilíbrio, ainda que com sinais de irregularidade (BERNASOL; LIMA-RIBEIRO, 20I0). Para Harper apud Felfili e Silva Júnior (1988), a maioria das florestas naturais não apresenta distribuição equilibrada, mas convergem para isto. Sendo assim, podemos afirmar que este cerrado apresenta padrão estrutural condizente com o observado para vegetações naturais não perturbadas, e inferir que, se não perturbado, é capaz de garantir o fluxo de indivíduos das primeiras classes para as últimas, mantendo a estrutura da comunidade independente da composição de espécies.

Em relação à distribuição das alturas constatou-

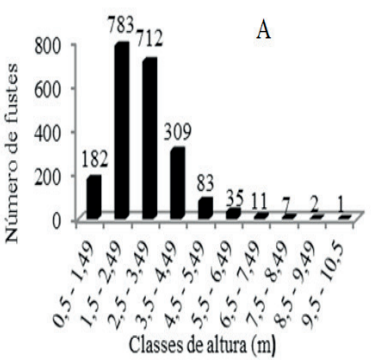

Classes de altura $(\mathrm{m})$

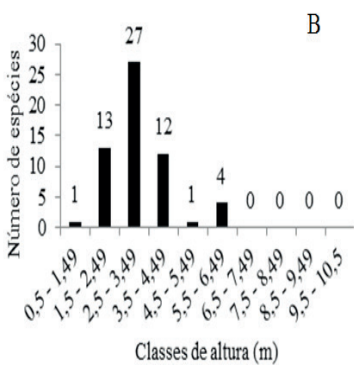

Classes de altura (m)
Figura 3 Distribuição das alturas no Cerrado sensu stricto de Jaborandi, $\mathrm{BA}$. $\mathrm{A}=$ número de fustes; $\mathrm{B}=$ número de espécies

Figure 3 Distribution of heights in the cerrado sensu stricto of Jaborandi, BA. $A=$ numbers of stems $=, B=$ number of species 
se uma vegetação marcada pela presença de indivíduos de pequenos porte. Cerca de $70 \%$ dos indivíduos apresentou altura entre I,5 e 3,5 metros (Figura 3A), padrão de distribuição comumente observado em Cerrado sensu stricto (ASSUNÇÃO; FELFILI, 2004). A altura máxima encontrada foi de $9,5 \mathrm{~m}$, correspondente a um indivíduo de Myrocarpus fastigiatus, responsável também pelo maior diâmetro registrado $(37 \mathrm{~cm})$. A altura mínima foi de $0,50 \mathrm{~m}$ registrada para indivíduos de Vellozia squamata. A altura média foi de 2,70 m (com desvio padrão de $\mathrm{Im}$ ) corroborando com Ribeiro e Walter (2008), ao afirmar que a altura média no Cerrado sensu stricto típico varia de 3 a 6 metros. Em relação às espécies, a maioria apresentou pequeno porte, em torno de 3 metros, sustentando mais uma vez as características típicas da estrutura desta fitofisionomia (Figura 3B) (FELFILI e SILVA JÚNIOR, 1988).

\section{CONCLUSÕES}

Os descritores fitossociológicos revelaram uma comunidade caracterizadas pela presença de poucas espécies dominantes, enriquecida principalmente pelo elevado número de espécies pouco comuns e raras. As estruturas diamétrica e hipsométrica evidenciaram uma vegetação equilibrada e compostas predominantemente por indivíduos de pequeno porte, onde a riqueza é relevante, assim como a diversidade local.

\section{AGRADECIMENTOS}

Agradecemos à Fazenda Guará pelo apoio logístico e pela valorização da biodiversidade do bioma Cerrado, e a engenheira florestal Renata Botelho Machado.

\section{REFERÊNCIAS BIBLIOGRÁFICAS}

APG. An update of the Angiosperm Phylogeny Group classification for the orders and families of flowering plants: APG III. Bot. J. Linn. Soc. I6I: I05-I2I. 2009.

ASSUNÇÃO, S. L.; FELFILI, J. M. Fitossociologia de um fragmento de cerrado sensu stricto na APA do Paranoá, DF, Brasil. Acta Botânica Brasílica I8(4): 903-909. 2004.

BERNASOL, W. P.; LIMA-RIBEIRO, M. S. Estrutura espacial e diamétrica de espécies arbóreas e seus condicionantes em um fragmento de cerrado sentido restrito no sudoeste goiano. Hoehnea, 37(2): I8I-198. 2010.

BRASIL. Ministério das Minas e Energia. Projeto RADAMBRASIL, Folha SD 23, Brasília: geologia, geomorfologia, pedologia, vegetação e uso da terra.
Rio de Janeiro, 1982. v. 29, 660 p.

CPRM. Relatório diagnóstico Sistema Aquífero Urucuia, Bacia Sedimentar Sanfranciscana / Companhia de Pesquisa de Recursos Minerais - Serviço Geológico do Brasil. Belo Horizonte. vol. I0, 43 p. 2012.

FELFILI, J. M.; EISENLOHR, P. V.; MELO, M. M. R. F. Procedimentos e Métodos de Amostragem da Vegetação. In: FELFILI J. M.; EISENLOHR, P. V.; MELO, M. M. R. F.; ANDRADE, L. A.; MEIRA NETO, J. A. A. Fitossociologia no Brasil. Viçosa: Editora UFV, 20I I. p. 86-I2I.

FELFILI, M. C.; Proposição de critérios florísticos, estruturais e de produção para o manejo de cerrado sensu stricto do Brasil central. 2008. 133p. Tese (Doutorado em Ciências Florestais) - Universidade de Brasília, Brasília, 2008.

FELFILI, J. M.; REZENDE, A. V.; SILVA-JÚNIOR, M. C. Biogeografia do Bioma Cerrado - Vegetação e solos da chapada dos Veadeiros. Brasília, Editora Universidade de Brasília, 2007. 242p.

FELFILI, J. M.; CARVALHO, F. A.; HAIDAR, R. F. Manual para o monitoramento da parcelas permanentes no biomas Cerrado e Pantanal. Brasília, Universidade de Brasília, Departamento de Engenharia Florestal, 2005. 55p.

FELFILI, J. M., SILVA JÚNIOR, M.C., SEVILHA, A.C., FAGG, C.W., WALTER, B.M.T., NOGUEIRA, P.E.; REZENDE, A.V. Diversity, floristics and structural patterns of cerrado vegetation in central Brazil. Plant Ecology, 75:37-46. 2004.

FELFILI, J.M., HARIDASSAM, M.; MENDONÇA, R.C.; FILGUEIRAS, T.S.; SILVA JÚNIOR, M.C; REZENDE, A. V. Projeto biogeografia do Bima Cerrado: Vegetação \& Solos. Caderno de Geociências do IBGE, Rio de Janeiro, n. I2: 75-166, out./dez. 1994.

FELFILI, J.M.; SILVA JÚNIOR, M.C. Distribuição dos diâmetros numa faixa de cerrado na Fazenda Água Limpa (FAL) em Brasília DF. Acta Botânica Brasílica, 2 (I-2): 85- 104. 1988.

HARIDASAN, M. Competição por nutrientes em espécies arbóreas do cerrado. In: SCARIOT, A.; SOUSA-SILVA, J.C.; FELFILI, J.M. Cerrado: ecologia, biodiversidade e conservação. Brasília: Ministério do Meio Ambiente. 2005. p. $167-178$.

HARPER, J. L. Population biology of plants. London, Academic Press. 1977. 892 p.

INEMA. Áreas prioritárias para conservação, uso sustentável e repartição dos benefícios da biodiversidade/ Instituto do Meio Ambiente e Recursos Hídricos - Governo do Estado da Bahia. 
Mapa temático. 2014.

KLINK. C, A. MACHADO. R, B. A conservação do Cerrado brasileiro. Megadiversidades, vol. I, n. I, p. I47-I55, Jul. 2005.

MACHADO, R. B.; AGUIAR, L. M. S.; CASTRO,A. A. J. F.; NOGUEIRA, C. C.; NETO, M. B. R. Caracterização da Fauna e Flora do Cerrado. In: FALEIRO, F; FARIAS NETO, A.L. Savanas - desafios e estratégias para o equilíbrio entre sociedade, agronegócio e recursos naturais. PlanaltinaDF: Embrapa Cerrados, 2008. p. 285-300.

MAguRRAM, A. E. Medindo a diversidade biológica. Paraná: Editora UFPR, 26 I p. $201 \mathrm{l}$.

MARIMON-JÚNIOR, B. H.; HARIDASAN, M. Comparação da vegetação arbórea e características edáficas de um cerradão e um cerrado sensu stricto em áreas adjacentes sobre solo distrófico no leste de Mato Grosso, Brasil. Acta Botânica Brasílica, v.19, n.4, p.9|3-926. 2005.

MEDEIROS, M. M.; FELFILI, J. M.; LIBANO, A. M. Comparação florístico-estrutural dos estratos de regeneração e adulto em cerrado sensu stricto no Brasil central. Cerne, Lavras, v. I3, n.3, p. 29I-298, jul./set. 2007.

MENDONÇA, R. C; FELFILI, J. M.; WALTER, B. M. T.; SILVA JÚNIOR, M. C.; REZENDE, A. V.; FILGUEIRAS, T. S.; NOGUEIRA, P. E.; FAGG, C. W. Flora Vascular do Bioma Cerrado. In: SANO, S. M.; ALMEIDA, S. P.; RIBEIRO, J. F. (Eds.). Cerrado: ecologia e flora. Brasília: Embrapa
Informação Tecnológica. 2008. p.42I-I.279.

MEYER, H. A. Structure, growth and grain in balance unevenaged forests. Journal of Forest. 50: 85 - 92. 1952.

MITTERMEIER, R. A.; GIL P. R.; HOFFMAN M.; PILGRIM J.; BROOKS T.; MITTERMEIER C. G.; LAMOREUX, J.; FONSECA G. A. B. (Eds.). Hotspots Revisited: Earth's Biologically Richest and Most Endangered Terrestrial Ecoregions. México: CEMEX. 2005. 392p.

RATTER, J. A.; BRIDGEWATER, S. RIBEIRO, J. F. Analysis of the floristic composition of the Brazilian Cerrado vegetation III: Comparison of the wood vegetation of 376 areas. Edinburg, Jornal of Botany, v. 60, p. 57- 109. 2003.

RESENDE, M. L. F; GUIMARÃES, L. L. Inventários da biogeografia do bioma Cerrado: Biogeografia de plantas. IBGE, I4p. 2007.

RIBEIRO, J. F.; WALTER, B. M. T. As Principais Fitofisionomias do Bioma Cerrado in: SANO, S. M.; DE ALMEIDA, S. P., RIBEIRO, J. F. Cerrado Ecologia e Flora. Embrapa Informação Tecnológica, Brasília, DF, Vol. I, 2008. 406 p.

SILVA, R. M.B. Relação Solo- Vegetação em uma Área de Cerrado sob Influência do Grupo Urucuia. 2011. 122p. Dissertação (Mestrado em Ciência Ambientais e Florestais). Universidade Federal Rural 